\title{
Oral PI3K- $\delta, \gamma$ Inhibitor for the Management of People with Chronic Lymphocytic Leukemia and Small Lymphocytic Lymphoma: A Narrative Review on Duvelisib
}

This article was published in the following Dove Press journal: OncoTargets and Therapy

\author{
Ankit Shah (D) \\ Jacqueline C Barrientos (D) ${ }^{2}$ \\ 'Division of Hematology-Oncology, \\ Department of Medicine at Rutgers New \\ Jersey Medical School, Newark, NJ, USA; \\ ${ }^{2}$ CLL Research and Treatment Center, \\ Division of Hematology-Oncology, \\ Department of Medicine at Zucker \\ School of Medicine at Hofstra/Northwell, \\ Hempstead, NY, USA
}

\begin{abstract}
The development of highly effective targeted therapies has led to a new treatment paradigm in patients with chronic lymphocytic leukemia or small lymphocytic lymphoma (CLL/SLL). Despite these advances, many patients will eventually require alternative treatment strategies due to the emergence of tolerability issues or resistance to these novel agents. Duvelisib is a first-in-class, potent oral agent with dual inhibitor activity against the $\delta$ and $\gamma$ isoforms of phosphoinositide 3-kinase (PI3K $\delta$ and $\mathrm{PI} 3 \mathrm{~K} \gamma$ ), which are specific to the hematopoietic system. Dysregulation of the PI3K/ PTEN/AKT/mTOR pathway has been implicated in cancer cell growth, survival and metabolism and has been the subject of cancer drug development in recent years. Duvelisib demonstrated activity in CLL/SLL in early trials, leading to further evaluation in the Phase 3 DUO trial that compared duvelisib against ofatumumab in patients with relapsed/refractory CLL/SLL. This trial led to the Food and Drug Administration (FDA) approval for the treatment of adult patients with CLL/SLL after at least two prior lines of therapy. The major reason for therapy discontinuation is the development of serious adverse events, which include severe infections and diarrhea/colitis, precluding its widespread use. Ongoing clinical trials are evaluating duvelisib in combination strategies and with alternate dosing schedules in patients with CLL/SLL. With close monitoring, duvelisib can be a promising drug for the treatment of patients with relapsed or refractory CLL/SLL. This review summarizes the relevant clinical data from recent clinical advances in CLL and aims to interpret the duvelisib trials while exploring strategies to improve its use and adverse event management in the era of novel targeted agents.
\end{abstract}

Keywords: duvelisib, CLL/SLL, PI3K, relapsed/refractory

\section{Introduction}

Chronic Lymphocytic Leukemia (CLL) and Small Lymphocytic Lymphoma (SLL) are considered to be different presentations of the same mature B-cell neoplasm. ${ }^{1,2}$ In 2020, the Surveillance, Epidemiology and End Result program (SEER) database estimated 21,040 new cases of CLL in the United States with 4060 deaths attributed to this disease. ${ }^{3}$ This translates into an incidence of CLL in the United States of approximately 5.0 per 100,000 men or women with a lifetime risk of $0.6 \%$. CLL is largely considered a disease of the elderly with a median age at diagnosis of 70 and
Correspondence: Jacqueline C Barrientos

Tel $+|-5| 6-470-4050$

Fax +I-516-470-4250

Email jbarrientos@northwell.edu 
affects men approximately twice as often as women. ${ }^{3}$ While several novel therapies have been developed for the treatment of CLL in recent years, most patients with CLL ultimately progress or relapse requiring therapy. ${ }^{4,5}$

The natural history of CLL/SLL is variable and outcomes are influenced by patient characteristics, clinical factors at the time of diagnosis, and the genetics of the tumor. Given the heterogeneity in clinical outcomes in CLL, not all patients require therapy at the time of diagnosis and clinical decision-making is centered on selecting optimal therapy taking into consideration the patient and the disease. Clinical factors associated with worse prognosis include advanced age, male sex, and clinical Rai or Binet stage. ${ }^{6}$ Genetic abnormalities associated with a poor prognosis in CLL include presence of del (11q), del (17p) or alterations in TP53 gene, and unmutated immunoglobulin heavy chain variable region (IGHV). ${ }^{7-13}$

\section{Current Treatment Approaches in CLL}

The majority of patients with a CLL diagnosis do not require therapy at the time of initial diagnosis and active surveillance is appropriate. The international workshop on CLL (iwCLL) has proposed criteria for the initiation of therapy and these recommendations highlight the importance of a treatment approach guided by symptoms or signs of disease progression. ${ }^{14}$ When therapy is indicated, there are several treatment options available for both the front-line and relapsed/refractory $(\mathrm{R} / \mathrm{R})$ setting. In the treatment-naive setting, in no particular order, continuous daily dosing with either Bruton's tyrosine kinase inhibitor (BTKi), ibrutinib or acalabrutinib, or fixed-duration therapy for 12 months with the BCL-2 inhibitor (BCL2i), venetoclax, given in combination with the monoclonal antibody obinutuzumab are Food and Drug Administration (FDA)-approved and preferred regimens for the treatment of CLL with or without del(17p).

Ibrutinib as monotherapy and in combination with both rituximab and obinutuzumab established efficacy in the treatment of CLL based on the findings of several clinical trials. ${ }^{15-21}$ One of the concerns is the potential toxicity that can occur as a result of indefinite treatment. The risk of atrial fibrillation along with the bleeding risk, require careful selection of candidates for this therapy. ${ }^{22}$ Furthermore, the risk of atrial fibrillation on ibrutinib therapy appears to be increased in patients with prior history of atrial fibrillation and age $>65$, the latter reflecting the majority of the overall CLL/SLL population. ${ }^{23}$ Importantly, there is a risk of developing hypertension over time, which can contribute to the risk for cardiac arrhythmias.

Acalabrutinib, a highly-selective, covalent, irreversible BTKi, has also received recent approval for the treatment of CLL based on initial findings from the ELEVATE-TN study and the ASCEND trial. In the ELEVATE-TN trial, both acalabrutinib monotherapy and acalabrutinib combined with obinutuzumab significantly prolonged median progression-free survival (PFS) compared to combination chlorambucil-obinutuzumab therapy in the front-line setting. ${ }^{24}$ In the ASCEND trial, acalabrutinib demonstrated superior PFS compared to investigator's choice of idelalisib or bendamustine in combination with rituximab in previously treated CLL/SLL patients. ${ }^{25}$ Together these two trials demonstrate the effectiveness of acalabrutinib for the treatment of CLL, however, similar to ibrutinibbased therapy, treatment with acalabrutinib carries a risk of atrial fibrillation $(\sim 3 \%)$ and bleeding (grade 3 or higher, $\sim 2 \%$ ) and requires continuous therapy until disease progression. ${ }^{24} \mathrm{~A}$ recent press release from the phase 3 ELEVATE R/R trial demonstrated that compared to ibrutinib, acalabrutinib met the study's primary endpoint of non-inferior PFS with statistically lower incidence of atrial fibrillation. ${ }^{26}$ Data from this trial are not yet available to determine how this will impact further guidance.

Venetoclax, a BCL2i, is also indicated for the first-line therapy of CLL in combination with obinutuzumab based on outcomes from the CLL14 trial, which showed a 3-year PFS of $81.9 \%{ }^{27,28}$ At a median follow-up of 39.6 months, the median PFS was not reached for the patients treated with venetoclax. A major advantage to this approach is that therapy is limited to 12 months, with the majority of patients being free of disease 2 years post completion of time-limited therapy. ${ }^{28}$ Given its mechanism of action of inhibiting the anti-apoptotic protein BCL-2, venetoclax use carries a risk of tumor lysis syndrome especially at the beginning and requires risk-adapted titration and monitoring per the manufacturer's guidelines. ${ }^{29}$

While chemo-immunotherapy (CIT) use has decreased in the front-line setting, fludarabine-cyclophosphamiderituximab (FCR) remains an option for select young, fit patients with mutated IGHV who do not have a $17 \mathrm{p}$ deletion or TP53 mutation. This finding is supported by long-term follow up from the original phase 2 single-arm study of FCR and from the phase 3 CLL8 trial which show that prolonged remissions, and potentially "cures", are possible. ${ }^{30-34}$ E1912 trial compared FCR against ibrutinib-rituximab in newly diagnosed CLL patients under the age of 70 without del 
(17p). Although patients treated with ibrutinib-rituximab had a superior PFS and overall survival (OS), in the subset of patients with mutated IGHV, there was no statistically significant difference in 3-years PFS. ${ }^{20}$ FCR, however, was associated with a higher rate of infectious complications, including neutropenic fever, and has been associated with an increased risk of treatment-related acute myeloid leukemia and myelodysplastic syndrome. ${ }^{20,35}$ While FCR has the advantages of fixed duration ( 6 cycles) and potential for cure, this needs to be weighed against the possible treatmentrelated toxicities when considering this regimen.

In the relapsed or refractory setting, there are several therapeutic options including any of the first-line regimens not previously administered, the $\mathrm{PI} 3 \mathrm{~K} \delta$ inhibitor, idelalisib, with or without rituximab, the $\mathrm{PI} 3 \mathrm{~K} \gamma, \delta$ inhibitor, duvelisib, and CIT. CIT use, however, is generally less favored in light of the availability of more effective and safer targeted agents such as an alternate tyrosine kinase inhibitor.

The optimal sequencing of therapies in the $\mathrm{R} / \mathrm{R}$ setting remains unknown at this time as randomized trials evaluating these novel agents after prior BTKi or BCL2 inhibitor therapy in the front-line setting are not available. Importantly, patients previously treated with BTKi, PI3Ki or BCL2 $\mathrm{i}$ were ineligible to participate in six landmark trials for relapsed/refractory disease with novel agents (RESONATE, ASCEND, M13-982, MURANO, Study 116, DUO). ${ }^{16,25,36-39}$ As a result, decisions regarding subsequent therapy require taking into consideration the patient's age and comorbidities, their response to prior therapies and the reasons for discontinuation (progression while on therapy vs relapse post completion of therapy vs intolerance), and the genetics of the CLL/SLL at the time of relapse (eg presence or absence of mutated TP53). ${ }^{40}$ Unfortunately, despite the panoply of options, patients with a CLL diagnosis may relapse making effective therapeutic options that target distinct pathways a necessity.

\section{Duvelisib: A First-in-Class PI3K- $\delta, \gamma$ Inhibitor for the Treatment of Relapsed/Refractory CLL}

The PI3K family of kinases plays an important role in intracellular signaling in response to growth factors, cytokines and chemokines, typically through activation of the $\mathrm{PI} 3 \mathrm{~K}$, protein kinase $\mathrm{B}$ (AKT), and mammalian target of rapamycin (mTOR) pathway. Deregulation of this pathway has been implicated in cancer cell growth, survival and metabolism and has been the subject of cancer drug development in recent years. Specifically, novel agents have been developed to target certain isoforms of Class I PI3K ( $\alpha, \beta, \gamma$ and $\delta$ ), which appears to be more integral to oncogenesis than PI3K classes II and III. ${ }^{41,42}$ While activating PI3KA mutations are often seen in solid tumor malignancies (eg $40 \%$ of hormone-receptor positive, HER2 negative metastatic breast cancers), these alterations are rarely seen in B-cell malignancies. ${ }^{41,43}$ Rather, PI3K activation in B-cell disorders occurs primarily through receptor tyrosine kinase-mediated signaling, such as via B-cell receptor (BCR) activation. While $\mathrm{PI} 3 \mathrm{~K} \alpha$ and $\mathrm{PI} 3 \mathrm{~K} \beta$ isoforms are ubiquitously expressed, $\mathrm{PI} 3 \mathrm{~K} \delta$ and $\mathrm{PI} 3 \mathrm{~K} \gamma$ are specific to the hematopoietic system. ${ }^{44}$ Pre-clinical studies have demonstrated that the PI3K $\delta$ isoform, which is selectively expressed by leukocytes, is crucial for B-cell survival and migration, making it an attractive target for therapies. PI3K $\gamma$, in contrast, is minimally expressed on B-cells, and instead is expressed on T-cells and myeloid cells. The rationale for targeting the $\gamma$ isoform of PI3K is that it may reduce cytokine production by T-cells and macrophages in the tumor microenvironment, which may be contributing to neoplastic B-cell survival. ${ }^{41,45,46}$

There are currently 4 FDA-approved PI3K inhibitors for the treatment of B-cell malignancies, idelalisib, copanlisib, duvelisib, and umbralisib. Idelalisib is an oral, highly selective inhibitor of $\mathrm{PI} 3 \mathrm{~K} \delta$, copanlisib is an intravenous pan-class I PI3K inhibitor with predominant activity against the $\mathrm{PI} 3 \mathrm{~K} \delta$ and $\mathrm{PI} 3 \mathrm{~K} \alpha$ isoforms, duvelisib (IPI$145)$ is a dual inhibitor against $\mathrm{PI} 3 \mathrm{~K} \delta$ and $\mathrm{PI} 3 \mathrm{~K} \gamma$, and umbralisib is a dual inhibitor of PI3K $\delta$ and CK $1 \varepsilon$. The inhibitory concentrations ( $\mathrm{IC}_{50}$ expressed in nanomolar) of these PI3K inhibitors are shown in Table 1.

Duvelisib's structure is similar to that of idelalisib, however, duvelisib has unique specificity (see Table 1) and

Table I IC 50 of Select PI3K Inhibitors Used in Hematologic Malignancies

\begin{tabular}{|l|l|l|l|l|l|}
\hline Drug & PI3K $\boldsymbol{\alpha}$ & $\mathbf{P I 3 K} \boldsymbol{\beta}$ & $\mathbf{P I 3 K \delta}$ & $\mathbf{P I 3 K} \boldsymbol{\gamma}$ & Reference \\
\hline Copanlisib & 0.5 & 3.7 & 0.7 & 6.4 & Liu $2013^{60}$ \\
\hline Duvelisib & 1602 & 84 & 2.5 & 27 & $\begin{array}{l}\text { Winkler } \\
2013^{47}\end{array}$ \\
\hline Idelalisib & 820 & 565 & 2.5 & 89 & $\begin{array}{l}\text { Lannutti } \\
2011^{61}\end{array}$ \\
\hline Umbralisib & $>10,000$ & 11116 & 22 & 1065 & $\begin{array}{l}\text { Burris } \\
2014^{62,63}\end{array}$ \\
\hline
\end{tabular}


pharmacokinetic properties including a longer target residence time. ${ }^{47,48}$ In pre-clinical studies, exposure of CLL cells activated by plate-bound anti-immunoglobulin $\mathrm{M}$ to duvelisib resulted in abrogation of BCR-mediated downstream AKT and ERK signaling, direct cytotoxicity to CLL B-cells, and diminished T-cell production of pro-survival cytokines $^{49,50}$ (Figure 1). In vitro, duvelisib appeared to successfully overcome resistance due to the point mutation BTK C481S, a frequently implicated resistance mechanism to ibrutinib therapy. Additionally, mouse models showed dual inhibition of PI3K $\delta$ and $\gamma$ isoforms resulted in a greater decrease in the number of CLL B-cells in the spleen compared to PI3K $\delta$ inhibition alone. ${ }^{51}$ These direct and indirect anti-neoplastic effects of duvelisib provided the basis for subsequent early-phase clinical trials in advanced hematologic malignancies.

\section{Clinical Efficacy of Duvelisib in CLL}

Duvelisib (Copiktra ${ }^{\circledR}$ ) is currently FDA-approved for the treatment of relapsed or refractory CLL/SLL or FL after at least two prior therapies and has been granted orphan drug designation for the treatment of T-cell lymphoma. Duvelisib was first studied in IPI-145-02 trial, a phase 1, open label, dose-escalation study in patients with advanced hematologic malignancies including indolent and aggressive non-Hodgkin's lymphoma (27\%), T-cell lymphoma (17\%), aggressive $R / R$ and treatment naive (TN) CLL (35\%), and other hematologic malignancies (21\%). ${ }^{52}$ In this study, the maximum tolerated dose was determined to be $75 \mathrm{mg}$ twice daily (BID) based on 2 dose-limiting toxicities occurring at the $100 \mathrm{mg}$ dose. Doses of $25 \mathrm{mg}$ or $75 \mathrm{mg}$ both resulted in a rapid inhibition of p-AKT with nearcomplete inhibition of CLL proliferation at the start of the second cycle of therapy. Furthermore, a subset of serum cytokine and chemokine levels associated with the B-cell tumor microenvironment were decreased following therapy exposure compared to baseline. The overall response rate (ORR) in patients with $R / R$ CLL and TN CLL was $56 \%$ and $83 \%$, respectively, with a median PFS of 15.7 months in the R/R cohort across all dose levels and not reached in the $\mathrm{TN}$ cohort who only received duvelisib $25 \mathrm{mg}$ BID. OS was not reached in either cohort. In subgroup analysis, duvelisib was

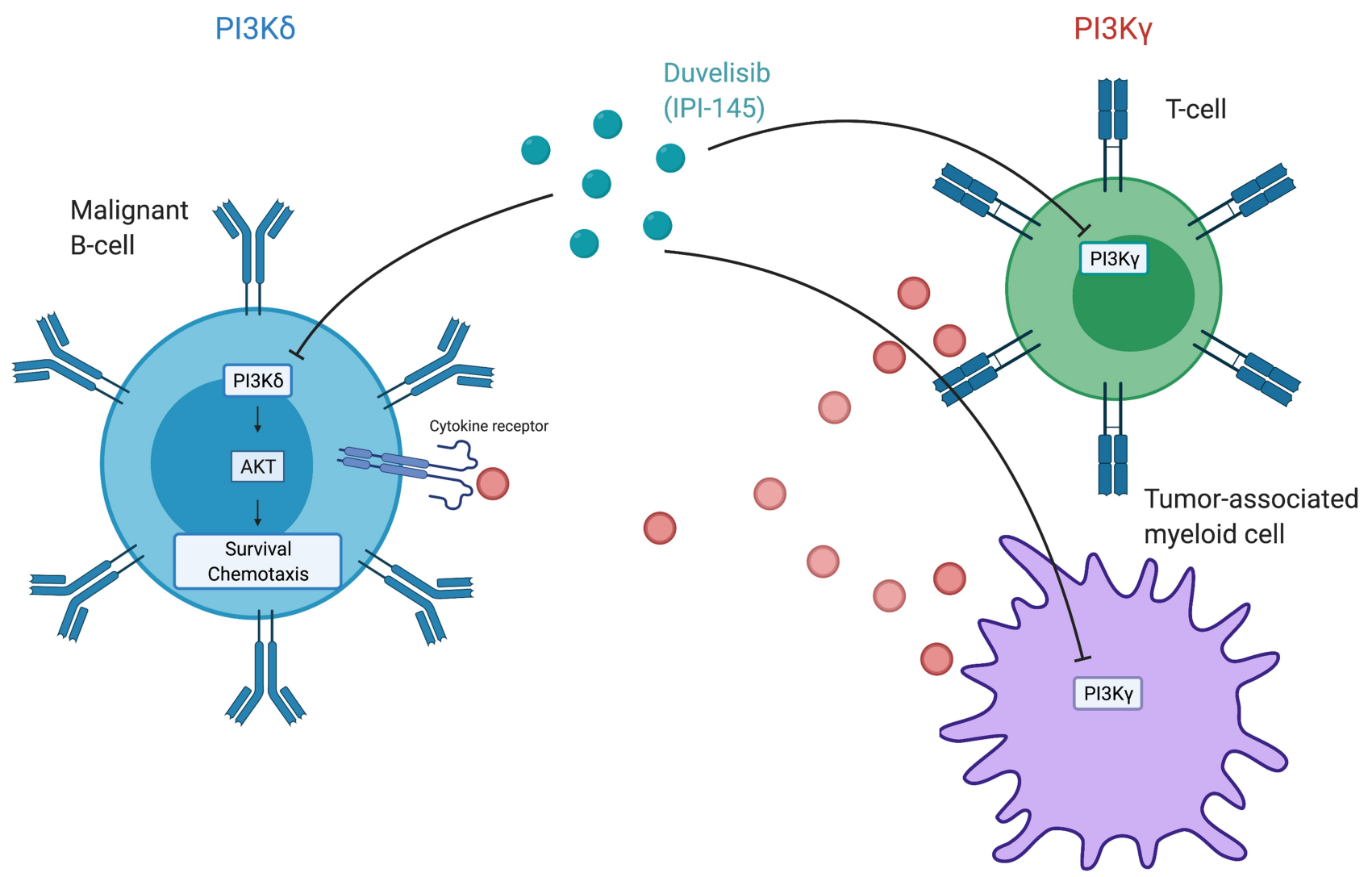

Figure I Duvelisib's activity on PI3K $\delta$ in malignant B-cells results in abrogation of down-stream signaling of the AKT/mTOR pathway thus inhibiting survival and chemotaxis. Duvelisib's activity on PI3K $\gamma$ results in decreased production of pro-survival cytokines from T-cells and tumor-associated myeloid cells. Created with BioRender.com. 
also efficacious in patients with high-risk biology such as those with $17 \mathrm{p}$ deletion or TP53 mutation (ORR = $46 \%)$ and those with unmutated IGHV (ORR $=51 \%)$. Given clinical activity at $25 \mathrm{mg}$ BID and no further suppression in pAKT and CLL proliferation at higher doses, this dose was selected for subsequent phase II and III studies. ${ }^{52,53}$

IPI 145-02 trial was followed by the phase 2 DYNAMO and phase 3 DUO trials. The DYNAMO trial enrolled patients with $\mathrm{R} / \mathrm{R}$ indolent non-Hodgkin's lymphoma (iNHL), including SLL, follicular lymphoma (FL) and marginal zone lymphoma (MZL), to receive duvelisib at a dose of $25 \mathrm{mg}$ BID. Patients had to have disease refractory to both rituximab and chemotherapy (including a purine analogue or alkylating agent) or radioimmunotherapy. Patients previously treated with BTKi or an alternate PI3K $\delta$ inhibitor were excluded from participation. In the overall treatment cohort the ORR was $47 \%$, meeting the pre-specified threshold of $30 \%$ as its primary endpoint. Notably, $22 \%$ of these patients had a diagnosis of SLL and in this subgroup the ORR was $68 \%$ by independent review committee (IRC) and $86 \%$ by investigator assessment. All of these responses were classified as partial by IRC. In the overall treatment cohort, the median PFS was 9.5 months with a median OS of 29 months. ${ }^{54}$ In the phase 3 DUO trial, patients with R/R CLL/SLL were randomized to receive duvelisib $25 \mathrm{mg}$ BID or ofatumumab. Patients had to have progressed after 1 prior line of therapy and could not have been previously treated with $\mathrm{BTK}$ or $\mathrm{PI} 3 \mathrm{~K} \delta$ inhibitor. With a median follow up of 22.4 months, treatment with duvelisib resulted in a superior PFS of 13.3 months compared with 9.9 months in the ofatumumab arm with this benefit extending to patients with del (17p) and TP53 mutations. By investigator assessment, median PFS in the duvelisib arm was 17.6 months vs 9.7 months in the ofatumumab. The ORR was also more favorable in the duvelisib arm (74\% vs 45\%). Similar to other B-cell receptor inhibitors, the majority were partial responses, with $85 \%$ of patients treated with duvelisib having a lymph node response (defined at $>50 \%$ decrease in the sum of the products of target lymph nodes). The median OS was not reached in either arm with $86 \%$ of patients alive at 12-months. ${ }^{36}$ The efficacy of duvelisib in these clinical trials is summarized in Table 2.

\section{Safety and Tolerability of Duvelisib in CLL}

In the phase 1, dose-escalation trial, IPI 145-02, serious AEs ( $\geq$ grade 3 ) occurred in $80 \%$ and $87 \%$ of patients who received duvelisib $25 \mathrm{mg}$ BID and $75 \mathrm{mg}$ BID, respectively. Hematologic toxicities including neutropenia, anemia and thrombocytopenia were common ( $\geq$ grade 3 , $32 \%$ ), however, treatment was rarely discontinued due to these events. The most common non-hematologic toxicities of any grade were diarrhea ( $42 \%$; $\geq$ grade $3,11 \%)$, fatigue $(41 \%$; $\geq$ grade $3,9 \%)$, elevated ALT $(38 \%$; $\geq$ grade $3,16 \%)$, elevated AST (38\%; $\geq$ grade $3,14 \%)$, and pyrexia $(35.2 \%$; $\geq$ grade $3,1.4 \%)$. The median duration of transaminase elevation was 2 weeks, with a median time of onset of 1.2 months. Elevated transaminases led to discontinuation of therapy in $7 \%$ of patients. The median time to onset of any grade diarrhea was 2 months with a median time to severe diarrhea ( $\geq$ grade 3 ) measured at $\sim 5$ months. Infectious complications occurred in $61 \%$ of patients, including 3 patients with Pneumocystis jirovecii pneumonia (PJP) and 2 patients with cytomegalovirus (CMV) infection. A protocol amendment in March 2013 required mandatory PJP prophylaxis in all study patients. In total, $27 \%$ of patients discontinued therapy at a dose of $25 \mathrm{mg}$ BID due to treatment-related AEs. ${ }^{52}$

In the treatment expansion cohort of study 145-02 which included 73 patients with R/R or TN CLL, the incidence of AEs was similar to other advanced hematologic malignancy subtypes. The most frequent non-hematologic toxicity, diarrhea $(47 \%$; $\geq$ grade $3,9 \%)$, led to discontinuation in two patients. Although transaminase elevation occurred in $31 \%$ ( $\geq$ grade $3,9 \%$ ) of patients, therapy was ultimately able to be restarted, often with dose-reductions. Infection occurred in $73 \%$ of $\mathrm{R} / \mathrm{R}$ patients, mostly presenting in the form of pneumonia $(38 \%)$ or upper respiratory tract infection $(27 \%)$. A total of $20 \mathrm{R} / \mathrm{R}$ patients $(36 \%)$ discontinued duvelisib due to AEs including the following which occurred in 2 or more patients: pneumonitis (4), pneumonia (2), stomatitis (2), diarrhea (2) and colitis (2). Two patients with R/R CLL died due to treatment-related infectious complications. Toxicities were overall similar in the TN cohort, with a higher incidence of grade 3 or higher diarrhea (22\%). In both the $\mathrm{R} / \mathrm{R}$ and $\mathrm{TN}$ cohorts, the most frequent reason for treatment discontinuation was AEs (36\% and 33\%, respectively). ${ }^{53}$ 
Table 2 Efficacy of Duvelisib in Clinical Trials

\begin{tabular}{|c|c|c|c|}
\hline Study & Regimen & Population & Efficacy Results \\
\hline $\begin{array}{l}\text { IPI I } 45-02^{52} \\
\text { Phase I dose escalation }\end{array}$ & Duvelisib 8-100mg BID & $\begin{array}{l}\text { Aggressive hematologic malignancies, } n= \\
210\end{array}$ & $\begin{array}{l}\text { iNHL: } \\
\text { ORR = } 58 \%, 6 \text { CRs } \\
\text { PFS = } 14.7 \mathrm{mo} . \\
\text { PTCL: } \\
\text { ORR = } 50 \%, 3 \mathrm{CR} \\
\text { TTR }=1.8 \mathrm{mo} .\end{array}$ \\
\hline $\begin{array}{l}\text { IPI I } 45-02^{53} \\
\text { R/R and } T N C L L \\
\text { Phase I expansion } \\
\text { cohort }\end{array}$ & $\begin{array}{l}\text { R/R CLL: duvelisib } 25-75 \mathrm{mg} \text { BID } \\
\text { TN CLL: duvelisib } 25 \mathrm{mg} \text { BID }\end{array}$ & $\begin{array}{l}\text { R/R CLL, } n=55 \\
T N C L L, n=18\end{array}$ & $\begin{array}{l}\text { R/R CLL: } \\
\text { ORR = 56\%, I CR } \\
\text { DOR = } 21 \mathrm{mo} . \\
\text { PFS = I5.7 mo. } \\
\text { OS = NR } \\
\text { TTR = } 1.9 \mathrm{mo} . \\
\text { TN CLL: } \\
\text { ORR = 83.3\%, O CR } \\
\text { DOR = NR } \\
\text { PFS = NR } \\
\text { OS = NR } \\
\text { TTR = } 3.7 \mathrm{mo} .\end{array}$ \\
\hline $\begin{array}{l}\text { DYNAMO }{ }^{54} \\
\text { Phase } 2\end{array}$ & Duvelisib 25mg BID & $\begin{array}{l}\text { Indolent NHL, } n=129 \\
\text { FL, } n=83 \\
\text { SLL, } n=28 \\
\text { MZL, } n=18\end{array}$ & $\begin{array}{l}\text { All patients: } \\
\text { ORR = 47.4\% } \\
\text { DOR = } 10 \mathrm{mo} . \\
\text { PFS = } 9.5 \mathrm{mo} . \\
\text { OS }=28.9 \mathrm{mo} . \\
\text { TTR }=1.9 \mathrm{mo} .\end{array}$ \\
\hline $\begin{array}{l}\text { DUO }^{36} \\
\text { Phase } 3\end{array}$ & $\begin{array}{l}\text { Duvelisib } 25 \mathrm{mg} \text { BID } \\
\text { Ofatumumab }\end{array}$ & $\begin{array}{l}\text { R/R CLL/SLL } \\
\text { Duvelisib, } n=160 \\
\text { Ofatumumab, } n=159\end{array}$ & $\begin{array}{l}\text { Duvelisib vs Ofatumumab: } \\
\text { PFS = I } 3.3 \text { vs } 9.9 \text { mo. }(\mathrm{HR}=0.52) \\
\text { ORR: } 74 \text { vs } 45 \% \\
\text { OS: NR (both arms) }\end{array}$ \\
\hline
\end{tabular}

Abbreviations: BID, twice daily; iNHL, indolent non-Hodgkin's lymphoma; ORR, overall response rate; CR, complete response; PFS, median progression-free survival; PTCL, peripheral T-cell lymphoma; TTR, time to response; R/R, relapsed/refractory; TN, treatment naïve; DOR, duration of response; OS, median overall survival; NR, not reached; FL, follicular lymphoma; MZL, marginal zone lymphoma; HR, hazard ratio.

In the phase 2 DYNAMO trial, which enrolled patients with iNHL, the most common AEs (all grades) were diarrhea $(49 \%)$, nausea $(29.5 \%)$, neutropenia $(29 \%)$, fatigue $(28 \%)$, and cough $(27 \%)$. The most common grade 3 or higher AEs included neutropenia (24.8\%), diarrhea $(15 \%)$, anemia (15\%), and thrombocytopenia (11\%). Dose interruptions or dose-reductions occurred in $70 \%$ of study patients. In this study, approximately one fourth (24\%) of patients discontinued therapy due to AEs and 5 deaths were considered treatment-related including pancolitis (1), fatal viral infection (1), septic shock (1), and severe skin toxicity (2). ${ }^{54}$

In patients who received duvelisib in the phase 3 DUO trial, adverse effects were similar to earlier studies with few notable differences. The rate of grade 3 or higher elevated AST or ALT was lower ( $3 \%$ each) than previously reported in the CLL expansion cohort of IPI 145-02 (14-$16 \%$ ), but similar to DYNAMO (3-5\%). Additionally, colitis emerged as a significant toxicity and was the most common immunomodulatory AEs of grade 3 or higher $(12 \%)$, resulting in treatment discontinuation in $5 \%$ of patients. In total, 4 deaths occurred due to AEs including staphylococcal pneumonia (2), sepsis (1) and general health deterioration (1). Similar to the expansion cohort of IPI-145-02 of R/R or TN CLL patients, the most common cause for treatment discontinuation were AEs (35\%), followed by progressive disease (22\%). ${ }^{36}$

Taken together, these studies highlight the major toxicities of duvelisib that require careful monitoring. These seem to be related to a class effect of PI3Ki and include 
immunomodulatory AEs, infectious complications, and hematologic toxicities. Appropriate careful monitoring allows for dose interruption or dose-reduction to minimize the risk of severe complications. Interestingly, the most frequent reason for treatment discontinuation remained AEs rather than disease progression in the CLL cohort. The findings in patients with a CLL diagnosis differs from those reported in DYNAMO where, in a study population of iNHL of which only a minority of patients had SLL $(21 \%)$, only one quarter of treatment discontinuations were due to AEs. ${ }^{36,52-54}$ This prompts to question whether there may be something unique to patients with a CLL/SLL diagnosis that leads to higher rates of AEs requiring discontinuation. Investigations are ongoing to evaluate the role of intermittent dosing of duvelisib to reduce toxicity in clinical trial NCT03961672. Preliminary data examining intermittent dosing after 2 cycles of continuous therapy with a selective PI3K $\delta$ inhibitor in development, ME-401 (Zandelisib), suggest this strategy may be effective in reducing rates of delayed immune mediated AEs without sacrificing efficacy. ${ }^{55}$

As a result of rare, but fatal, events seen in clinical trials to date, duvelisib carries black-box warnings for serious infections, severe diarrhea or colitis, serious cutaneous reactions and pneumonitis. These toxicities need to be monitored for and the manufacturer has provided guidance regarding the management of these AEs depending on their severity. All patients on duvelisib should receive infection prophylaxis for PJP and CMV when initiating duvelisib; along with monthly CMV viral load monitoring. It is recommended to withhold duvelisib until any new infection resolves and to discontinue its use if PJP is confirmed. For immunologic toxicities such as diarrhea/ colitis, pneumonitis, or rash, we recommend holding duvelisib and prompt initiation of steroid therapy along with supportive care when infectious causes have been ruled out, with permanent discontinuation in cases of grade 3 or higher AEs. Regarding transaminitis, patients are usually asymptomatic, hence the need to monitor liver enzymes during routine medical care and to hold further dosing until the transaminitis resolves or decreases to grade 1 toxicity. For hematological toxicities (including neutropenia and thrombocytopenia), duvelisib should be withheld when the absolute neutrophil count falls below $500 / \mathrm{mm}^{3}$ (grade 4) or the platelet count drops below $25,000 / \mathrm{mm}^{3}$ (grade 4).

It is important to evaluate potential drug-drug interactions to minimize the risk of under- or over-dosing. Co- administration of duvelisib with strong CYP3A4 inducers may decrease the efficacy of the drug and should be avoided. When co-administered with strong or moderate CYP3A inhibitors, duvelisib should be dosed at $15 \mathrm{mg}$ twice daily and clinicians should monitor carefully for potential toxicities.

\section{Comparing Duvelisib to Idelalisib for the Treatment of Relapsed/ Refractory CLL/SLL}

As discussed earlier, idelalisib (GS-1101 or CAL-101) is an oral, highly selective inhibitor of $\mathrm{PI} 3 \mathrm{~K} \delta$ and is the first PI3K inhibitor to be FDA approved for the treatment of CLL/SLL. Idelalisib's approval was based on the results from Study 116, a phase 3 randomized control trial comparing idelalisib with rituximab vs rituximab monotherapy in patients unfit to receive cytotoxic therapy. The median number of prior therapies in this trial was 3 and prior therapy had to include a CD20 antibody or at least two prior cytotoxic regimens. PFS at 24 weeks was $93 \%$ in the idelalisib group vs $46 \%$ in the rituximab group. OS at 12 months was also superior in the idelalisib-treated arm $(92 \%$ vs $80 \%){ }^{38}$ With longer follow up the median PFS in patients randomized to receive idelalisib was 20.3 months. ${ }^{56}$

There have been no clinical trials directly comparing duvelisib and idelalisib to date. Given that there are differences in the clinical trials leading to each drug's approval, cross-trial comparisons are not possible. Table 3 summarizes patient characteristics, outcomes and specific AEs (those of interest that were reported comparably) in DUO and Study $116 .{ }^{36,56}$ At present, due to lack of direct comparison, and differences in trial population and followup, we are unable to recommend one PI3K inhibitor over another and believe both to be reasonable options if PI3K inhibition is the selected therapeutic approach.

\section{Future Considerations}

There are currently 3 registered, ongoing, clinical trials utilizing duvelisib in CLL/SLL (Table 4; clinicaltrials. gov). As discussed previously, the number one reason for discontinuation of therapy in patients with CLL is AEs rather than progressive disease. ${ }^{36,53}$ While prophylaxis for PJP and CMV with careful monitoring for infection may mitigate some of the potentially fatal toxicities of duvelisib, further study is needed to mitigate the immunomodulatory side effects including 
Table 3 Patient Characteristics and Outcomes in DUO and Study 116

\begin{tabular}{|c|c|c|}
\hline & $\begin{array}{l}\text { Duvelisib Arm } \\
\text { (DUO) }^{36}\end{array}$ & $\begin{array}{l}\text { Idelalisib Arm } \\
\text { (Study } 116)^{56}\end{array}$ \\
\hline \multicolumn{3}{|c|}{ Patient demographics and characteristics of interest } \\
\hline Median age & 69 & 71 \\
\hline $\begin{array}{l}\operatorname{del}(17 \mathrm{p}) \text { or TP53 } \\
\text { mutation }(\%)\end{array}$ & 31 & 43.2 \\
\hline IGHV unmutated (\%) & 69 & 83.6 \\
\hline $\begin{array}{l}\text { Median prior lines of } \\
\text { therapy }\end{array}$ & 2 & 3 \\
\hline \multicolumn{3}{|l|}{ Patient outcomes } \\
\hline ORR (\%) & 73.8 & 85.5 \\
\hline Median PFS (mo.) & 13.3 & 20.3 \\
\hline $\begin{array}{l}\text { Overall Survival } \\
\text { (mo.) }\end{array}$ & NR & 40.6 \\
\hline $\begin{array}{l}\text { Duration of exposure } \\
\text { (mo.) }\end{array}$ & 11.5 & 16.2 \\
\hline \multicolumn{3}{|c|}{$\begin{array}{l}\text { Incidence of AEs of interest; \% all grades (\% grade } 3 \text { and } \\
\text { above) }\end{array}$} \\
\hline Any AE & $99(87)$ & $98.2(90.9)$ \\
\hline Diarrhea & $51(15)$ & $46.4(16.4)$ \\
\hline Colitis & $13(12)$ & $10.9(8.2)$ \\
\hline Pyrexia & $29(3)$ & $47.3(3.6)$ \\
\hline Death due to $\mathrm{AE}$ & 11.9 & 11.8 \\
\hline
\end{tabular}

Abbreviations: ORR, overall response rate; PFS, progression-free survival; $A E$, adverse event; NR, not reported.

diarrhea, colitis, pneumonitis and rash. One potential solution may be to develop new dosing schedules. In the phase 2 trial NCT03961672, investigators are evaluating the role of a 12-week (3-cycle) induction phase where patients receive continuous therapy with duvelisib $25 \mathrm{~m}$ BID, followed by a maintenance phase involving duvelisib dosing on days $1-2,8-9,15-16$ and $22-23$ of each 28-day cycle or the first 2 days of each week. As the median time to severe diarrhea in IPI-145-02 was 5 months, by altering dosing after 12 weeks, one may be able to mitigate the development of this $\mathrm{AE}$ while still effectively targeting PI3K- $\delta, \gamma{ }^{53}$

Pre-clinical studies have shown that duvelisib can overcome ibrutinib resistance mediated by the in BTK C481S mutation, however, there is very limited data regarding the
Table 4 Ongoing Trials of Duvelisib in CLL/SLL

\begin{tabular}{|l|l|l|l|}
\hline Study & Regimen & Population & $\begin{array}{l}\text { Target Accrual/ } \\
\text { Primary } \\
\text { Endpoint(s) }\end{array}$ \\
\hline $\begin{array}{l}\text { NCT0396I672 } \\
\text { Phase 2 }\end{array}$ & $\begin{array}{l}\text { Intermittent } \\
\text { duvelisib }\end{array}$ & $\begin{array}{l}\text { R/R CLL/ } \\
\text { SLL }\end{array}$ & $\begin{array}{l}\mathrm{N}=30 \\
\text { Endpoint: PFS }\end{array}$ \\
\hline $\begin{array}{l}\text { NCT0420962I } \\
\text { Phase 2 }\end{array}$ & $\begin{array}{l}\text { Duvelisib } \\
\text { overlapping } \\
\text { with } \\
\text { ibrutinib } \\
\text { (initial 6 mo.) }\end{array}$ & $\begin{array}{l}\text { Ibrutinib- } \\
\text { resistant } \\
\text { CLL/SLL } \\
\text { patients }\end{array}$ & $\begin{array}{l}\mathrm{N}=36 \\
\text { Endpoint: ORR }\end{array}$ \\
\hline $\begin{array}{l}\text { NCT03534323 } \\
\text { Phase I/2 }\end{array}$ & $\begin{array}{l}\text { Duvelisib + } \\
\text { venetoclax }\end{array}$ & $\begin{array}{l}\text { CLL/SLL or } \\
\text { RS }\end{array}$ & $\begin{array}{l}\mathrm{N}=67 \\
\text { Endpoint(s): MTD } \\
\text { of venetoclax } \\
\text { when administered } \\
\text { with duvelisib, CR } \\
\text { rate }\end{array}$ \\
\hline
\end{tabular}

Abbreviations: PFS, median progression-free survival; ORR, overall response rate; RS, Richter syndrome; MTD, maximum tolerated dose; CR, complete response.

efficacy of duvelisib after BTKi therapy in human subjects. ${ }^{50}$ Very few patients (6) had received prior BTKi therapy in IPI$145-02$ and in the phase 3 DUO trial patients with prior BTKi therapy were excluded, therefore clinical data regarding the efficacy of duvelisib in ibrutinib-resistant and ibrutinibintolerant patients are lacking. ${ }^{36,53}$ In the ongoing trial, NCT04209621, investigators are evaluating the use of duvelisib after development of ibrutinib resistance and hopefully more data will be forthcoming. As patients may have rapid progression upon abrupt discontinuation of ibrutinib, BTKi therapy in this trial is overlapped for 6 months while initiating duvelisib to prevent a potential "rebound" effect. Recruitment status was suspended, however, on September 24, 2020 due to an unexpected sudden death on study.

Lastly, in NCT03534323, duvelisib is being studied in combination with venetoclax for the treatment of patients with CLL who had progressed after 1 prior therapy and those with Richter's transformation. Preclinical studies in CLL/SLL have shown that duvelisib therapy results in upregulation of the anti-apoptotic protein BCL2. Furthermore, combination duvelisib and venetoclax therapy in vitro, resulted in more apoptosis than venetoclax therapy alone. ${ }^{57}$ These findings provide the rationale for this combination in early clinical trials. If efficacious, tolerable and safe, a follow up study will be prudent to 
assess whether this therapy can be given for a fixed duration similar to venetoclax plus obinutuzumab.

An important, practical question is when to employ PI3K inhibitor therapy in the treatment of CLL/SLL. Expert consensus is generally to utilize BTKi and venetoclax therapy in first and second lines of therapy, in no specific order, as these are felt to be the most active agents. ${ }^{58}$ Patients exposed to both these agents were generally excluded, however, from trials involving duvelisib, therefore efficacy is largely extrapolated in this population. Retrospective data from Mato et al. suggest that patients who are refractory to both BTKi and venetoclax appear to have a short duration of response with PI3K inhibitor therapy. ${ }^{59}$ For these high-risk patients we recommend enrollment in a clinical trial or evaluation for cellular therapies (e.g chimeric antigen receptor T-cell therapy or allogeneic stem cell transplant) when possible. In the absence of these options, most would offer PI3K inhibitor therapy given the lack of other more effective, commercially available, targeted agents. At the moment, patients with del(17p) or double refractory to BTKi and BCL2i remain an unmet need and may benefit from duvelisib salvage therapy in the absence of a clinical trial.

\section{Conclusions}

In recent years, several novel agents have been developed for the treatment of CLL/SLL. Unfortunately, due to the relapsing nature of the disease, treatment options targeting different signaling pathways are still needed in order to prolong survival. By inhibiting the $\gamma$ and $\delta$ isoforms of PI3K, duvelisib targets key oncogenic pathways resulting in direct cytotoxic effects on tumor cells and abrogation of pro-survival cytokine and chemokine signaling in the tumor microenvironment. ${ }^{41}$ Duvelisib has demonstrated efficacy in CLL/SLL based on the Phase 1 IPI $145-02$ and phase 3 DUO trials and has received FDA approval in patients with R/R CLL/SLL after 2 prior therapies. One of the obstacles to duvelisib therapy, however, is its safety profile, which leads to treatment discontinuation in the majority of cases. $^{36,53}$ Consequently, several clinical trials are investigating alternative dosing schedules to reduce toxicities while maintaining efficacy. The efficacy of duvelisib in CLL is also being studied in ibrutinib-resistant patients and in combination with other targeted agents such as venetoclax. The results of these clinical trials will help to better define the role of duvelisib in the growing CLL/SLL armamentarium. Overall, duvelisib represents a promising targeted agent with an evolving role in the treatment of relapsed/refractory CLL/SLL.

\section{Acknowledgments}

Jacqueline C. Barrientos' work is supported in part by the 2015 American Society of Hematology-Harold Amos Medical Faculty Development Program fellowship and the 2019 Paul Foundation Innovation Award.

\section{Disclosure}

JCB has received honoraria for consulting for Gilead, AstraZeneca, Janssen, Genentech, Abbvie and research support from Pharmacyclics/Abbvie, AstraZeneca, and Gilead. The authors report no other conflicts of interest in this work.

\section{References}

1. Swerdlow SH, Campo E, Pileri SA, et al. The 2016 revision of the World Health Organization classification of lymphoid neoplasms. Blood. 2016;127(20):2375-2390. doi:10.1182/blood-2016-01-643569

2. World Health Organization. Pathology and Genetics of Tumours of Haematopoietic and Lymphoid Tissues. IARC; 2001.

3. Chiorazzi N, Chen SS, Rai KR. Chronic lymphocytic leukemia cancer stat facts. Cold Spring Harb Perspect Med. 2021;11(2): a035220. doi:10.1101/cshperspect.a035220

4. Weide R, Feiten S, Chakupurakal G, et al. Survival improvement of patients with chronic lymphocytic leukemia (CLL) in routine care 1995-2017. Leuk Lymphoma. 2019;61(3):557-566. doi:10.1080/ 10428194.2019.1680840

5. Patel K, Danilov AV, Pagel JM. Duvelisib for CLL/SLL and follicular non-Hodgkin lymphoma. Blood. 2019;134(19):1573-1577. doi:10.1182/blood.2019001795

6. Pflug N, Bahlo J, Shanafelt TD, et al. Development of a comprehensive prognostic index for patients with chronic lymphocytic leukemia. Blood. 2014;124(1):49-62. doi:10.1182/blood-201402-556399

7. Döhner H, Stilgenbauer S, Benner A, et al. Genomic aberrations and survival in chronic lymphocytic leukemia. $N$ Engl J Med. 2000;343 (26):1910-1916. doi:10.1056/NEJM200012283432602

8. Damle RN, Wasil T, Fais F, et al. Ig V gene mutation status and CD38 expression as novel prognostic indicators in chronic lymphocytic leukemia. Blood. 1999;94(6):1840-1847. doi:10.1182/blood. V94.6.1840

9. Kröber A, Seiler T, Benner A, et al. VH mutation status, CD38 expression level, genomic aberrations, and survival in chronic lymphocytic leukemia. Blood. 2002;100(4):1410-1416. doi:10.1182/ blood.V100.4.1410.h81602001410_1410_1416

10. Rassenti LZ, Jain S, Keating MJ, et al. Relative value of ZAP-70, $\mathrm{CD} 38$, and immunoglobulin mutation status in predicting aggressive disease in chronic lymphocytic leukemia. Blood. 2008;112 (5):1923-1930. doi:10.1182/blood-2007-05-092882

11. Zenz T, Eichhorst B, Busch R, et al. TP53 mutation and survival in chronic lymphocytic leukemia. J Clin Oncol. 2010;28 (29):4473-4479. doi:10.1200/JCO.2009.27.8762

12. Rossi D, Cerri M, Deambrogi C, et al. The prognostic value of TP53 mutations in chronic lymphocytic leukemia is independent of Del17p13: implications for overall survival and chemorefractoriness. Clin Cancer Res. 2009;15(3):995-1004. doi:10.1158/1078-0432.CCR08-1630 
13. Rossi D, Rasi S, Spina V, et al. Integrated mutational and cytogenetic analysis identifies new prognostic subgroups in chronic lymphocytic leukemia. Blood. 2013;121(8):1403-1412. doi:10.1182/blood-201209-458265

14. Hallek M, Cheson BD, Catovsky D, et al. iwCLL guidelines for diagnosis, indications for treatment, response assessment, and supportive management of CLL. Blood. 2018;131(25):2745-2760. doi:10.1182/blood-2017-09-806398

15. Burger JA, Tedeschi A, Barr PM, et al. Ibrutinib as initial therapy for patients with chronic lymphocytic leukemia. $N$ Engl J Med. 2015;373 (25):2425-2437. doi:10.1056/NEJMoa1509388

16. Byrd JC, Brown JR, O'Brien S, et al. Ibrutinib versus ofatumumab in previously treated chronic lymphoid leukemia. $N$ Engl J Med. 2014;371(3):213-223. doi:10.1056/NEJMoa1400376

17. Farooqui MZH, Valdez J, Martyr S, et al. Ibrutinib for previously untreated and relapsed or refractory chronic lymphocytic leukaemia with TP53 aberrations: a phase 2, single-arm trial. Lancet Oncol. 2015;16(2):169-176. doi:10.1016/S1470-2045(14)71182-9

18. Moreno C, Greil R, Demirkan F, et al. Ibrutinib plus obinutuzumab versus chlorambucil plus obinutuzumab in first-line treatment of chronic lymphocytic leukaemia (iLLUMINATE): a multicentre, randomised, open-label, phase 3 trial. Lancet Oncol. 2019;20(1):43-56. doi:10.1016/S1470-2045(18)30788-5

19. O'Brien S, Jones JA, Coutre SE, et al. Ibrutinib for patients with relapsed or refractory chronic lymphocytic leukaemia with $17 \mathrm{p}$ deletion (RESONATE-17): a phase 2, open-label, multicentre study. Lancet Oncol. 2016;17(10):1409-1418. doi:10.1016/S1470-2045(16)30212-1

20. Shanafelt TD, Wang XV, Kay NE, et al. Ibrutinib-rituximab or chemoimmunotherapy for chronic lymphocytic leukemia. $N$ Engl $J$ Med. 2019;381(5):432-443. doi:10.1056/NEJMoa1817073

21. Byrd JC, Furman RR, Coutre SE, et al. Targeting BTK with ibrutinib in relapsed chronic lymphocytic leukemia. N Engl J Med. 2013;369 (1):32-42. doi:10.1056/NEJMoa1215637

22. Munir T, Brown JR, O'Brien S, et al. Final analysis from RESONATE: up to six years of follow-up on ibrutinib in patients with previously treated chronic lymphocytic leukemia or small lymphocytic lymphoma. Am J Hematol. 2019;94(12):1353-1363. doi:10.1002/ajh.25638

23. Brown JR, Moslehi J, O'Brien S, et al. Characterization of atrial fibrillation adverse events reported in ibrutinib randomized controlled registration trials. Haematologica. 2017;102(10):1796-1805. doi:10.3324/haematol.2017.171041

24. Sharman JP, Banerji V, Fogliatto LM, et al. ELEVATE TN: phase 3 study of acalabrutinib combined with obinutuzumab $(\mathrm{O})$ or alone vs O plus chlorambucil $(\mathrm{Clb})$ in patients (Pts) with treatment-naive chronic lymphocytic leukemia (CLL). Blood. 2019;134 (Supplement 1):31. doi:10.1182/blood-2019-128404

25. Ghia P, Pluta A, Wach M, et al. Acalabrutinib versus investigator's choice of idelalisib plus rituximab or bendamustine plus rituximab in relapsed or refractory CLL: the randomised, controlled, phase 3 ASCEND; 2020. Available from: https://papers.ssrn.com/sol3/ papers.cfm?abstract_id=3463285. Accessed February 19, 2020.

26. Calquence met primary efficacy endpoint in head-to-head trial against ibrutinib in chronic lymphocytic leukaemia; 2021. Available from: https://www.astrazeneca.com/media-centre/press-releases/2021/cal quence-met-primary-endpoint-against-ibrutinib.html. Accessed February 24, 2021.

27. Fischer K, Al-Sawaf O, Bahlo J, et al. Venetoclax and obinutuzumab in patients with CLL and coexisting conditions. $N$ Engl J Med. 2019;380(23):2225-2236. doi:10.1056/NEJMoa1815281

28. Al-Sawaf O, Zhang C, Tandon M, et al. Venetoclax plus obinutuzumab versus chlorambucil plus obinutuzumab for previously untreated chronic lymphocytic leukaemia (CLL14): follow-up results from a multicentre, open-label, randomised, phase 3 trial. Lancet Oncol. 2020;21(9):1188-1200. doi:10.1016/S14702045(20)30443-5
29. AbbVie Inc. VENCLEXTA ${ }^{\mathrm{TM}}$ (venetoclax) [package insert]. U.S. Food and Drug Administration website. Available from: https:/www. accessdata.fda.gov/drugsatfda_docs/label/2016/208573s000lbl.pdf. Accessed February 20, 2021.

30. Tam CS, O'Brien S, Wierda W, et al. Long-term results of the fludarabine, cyclophosphamide, and rituximab regimen as initial therapy of chronic lymphocytic leukemia. Blood. 2008;112 (4):975-980. doi:10.1182/blood-2008-02-140582

31. Keating MJ, O'Brien S, Albitar M, et al. Early results of a chemoimmunotherapy regimen of fludarabine, cyclophosphamide, and rituximab as initial therapy for chronic lymphocytic leukemia. J Clin Oncol. 2005;23(18):4079-4088. doi:10.1200/ JCO.2005.12.051

32. Thompson PA, Tam CS, O'Brien SM, et al. Fludarabine, cyclophosphamide, and rituximab treatment achieves long-term disease-free survival in IGHV-mutated chronic lymphocytic leukemia. Blood. 2016;127(3):303-309. doi:10.1182/blood-2015-09-667675

33. Fischer K, Bahlo J, Fink AM, et al. Long-term remissions after FCR chemoimmunotherapy in previously untreated patients with CLL: updated results of the CLL8 trial. Blood. 2016;127(2):208-215. doi:10.1182/blood-2015-06-651125

34. Woyach JA. FCR holds up to the test of time: CLL8 follow-up. Blood. 2016;127(2):172-173. doi:10.1182/blood-2015-11-678557

35. Benjamini O, Jain P, Trinh L, et al. Second cancers in patients with chronic lymphocytic leukemia who received frontline fludarabine, cyclophosphamide and rituximab therapy: distribution and clinical outcomes. Leuk Lymphoma. 2015;56(6):1643-1650. doi:10.3109/ 10428194.2014.957203

36. Flinn IW, Hillmen P, Montillo M, et al. The phase 3 DUO trial: duvelisib vs ofatumumab in relapsed and refractory CLL/SLL. Blood. 2018;132(23):2446-2455. doi:10.1182/blood-2018-05850461

37. Seymour JF, Kipps TJ, Eichhorst B, et al. Venetoclax-rituximab in relapsed or refractory chronic lymphocytic leukemia. $N$ Engl J Med. 2018;378(12):1107-1120. doi:10.1056/NEJMoa1713976

38. Furman RR, Sharman JP, Coutre SE, et al. Idelalisib and rituximab in relapsed chronic lymphocytic leukemia. $N$ Engl J Med. 2014;370 (11):997-1007. doi:10.1056/NEJMoa1315226

39. Stilgenbauer S, Eichhorst B, Schetelig J, et al. Venetoclax in relapsed or refractory chronic lymphocytic leukaemia with $17 \mathrm{p}$ deletion: a multicentre, open-label, phase 2 study. Lancet Oncol. 2016;17 (6):768-778. doi:10.1016/S1470-2045(16)30019-5

40. Barrientos JC. Sequencing of chronic lymphocytic leukemia therapies. Hematol Am Soc Hematol Educ Program. 2016;2016 (1):128-136. doi:10.1182/asheducation-2016.1.128

41. Fruman DA. Targeting PI3K-gamma in non-hodgkin lymphoma. J Clin Oncol. 2019;37(11):932-934. doi:10.1200/JCO.19.00215

42. Engelman JA. Targeting PI3K signalling in cancer: opportunities, challenges and limitations. Nat Rev Cancer. 2009;9(8):550-562. doi: $10.1038 / \mathrm{nrc} 2664$

43. Mollon L, Aguilar A, Anderson E, et al. Abstract 1207: a systematic literature review of the prevalence of PIK3CA mutations and mutation hotspots in HR+/HER2- metastatic breast cancer. Cancer Res. 2018;78 (13Supplement):1207. doi:10.1158/1538-7445.AM2018-1207

44. Vangapandu HV, Jain N, Gandhi V. Duvelisib: a phosphoinositide3 kinase $\delta / \gamma$ inhibitor for chronic lymphocytic leukemia. Expert Opin Investig Drugs. 2017;26(5):625-632. doi:10.1080/ 13543784.2017.1312338

45. Barrientos JC. Idelalisib for the treatment of indolent non-Hodgkin lymphoma: a review of its clinical potential. OncoTargets Ther. 2016;9:2945-2953. doi:10.2147/OTT.S102573

46. Frustaci AM, Tedeschi A, Deodato M, Zamprogna G, Cairoli R, Montillo M. Duvelisib for the treatment of chronic lymphocytic leukemia. Expert Opin Pharmacother. 2020;21(11):1299-1309. doi:10.1080/14656566.2020.1751123 
47. Winkler DG, Faia KL, DiNitto JP, et al. PI3K- $\delta$ and PI3K- $\gamma$ inhibition by IPI-145 abrogates immune responses and suppresses activity in autoimmune and inflammatory disease models. Chem Biol. 2013;20 (11):1364-1374. doi:10.1016/j.chembiol.2013.09.017

48. Rodrigues DA, Sagrillo FS, Fraga CAM. Duvelisib: a 2018 novel FDA-approved small molecule inhibiting phosphoinositide 3-kinases. Pharmaceuticals. 2019;12(2):69. doi:10.3390/ph12020069

49. Balakrishnan K, Peluso $\mathrm{M}$, Fu $\mathrm{M}$, et al. The phosphoinositide-3-kinase (PI3K)-delta and gamma inhibitor, IPI-145 (Duvelisib), overcomes signals from the PI3K/AKT/S6 pathway and promotes apoptosis in CLL. Leukemia. 2015;29 (9):1811-1822. doi:10.1038/leu.2015.105

50. Dong S, Guinn D, Dubovsky JA, et al. IPI-145 antagonizes intrinsic and extrinsic survival signals in chronic lymphocytic leukemia cells. Blood. 2014;124(24):3583-3586. doi:10.1182/blood-2014-07-587279

51. Chen -S-S, Kutok JL, Ferrer G, et al. Dual inhibition of PI3K- $\delta$ and PI3K- $\gamma$ by duvelisib eliminates CLL B cells, impairs CLL-supporting cells, and overcomes ibrutinib resistance in a patient-derived xenograft model. Blood. 2018;132(Supplement 1):4420. doi:10.1182/ blood-2018-99-109853

52. Flinn IW, O’Brien S, Kahl B, et al. Duvelisib, a novel oral dual inhibitor of PI3K- $\delta, \gamma$, is clinically active in advanced hematologic malignancies. Blood. 2018;131(8):877-887. doi:10.1182/blood-201705-786566

53. O’Brien S, Patel M, Kahl BS, et al. Duvelisib, an oral dual PI3K- $\delta, \gamma$ inhibitor, shows clinical and pharmacodynamic activity in chronic lymphocytic leukemia and small lymphocytic lymphoma in a phase 1 study. Am J Hematol. 2018;93(11):1318-1326. doi:10.1002/ajh.25243

54. Flinn IW, Miller CB, Ardeshna KM, et al. DYNAMO: a phase II study of duvelisib (IPI-145) in patients with refractory indolent non-hodgkin lymphoma. J Clin Oncol. 2020.

55. Zelenetz AD, Soumerai JD, Jagadeesh D, et al. Preliminary safety and efficacy results with an intermittent schedule of the PI3k $\delta$ inhibitor ME-401 alone or in combination with rituximab for B-cell malignancies. Blood. 2018;132(Supplement1):2893. doi:10.1182/ blood-2018-99-115670
56. Sharman JP, Coutre SE, Furman RR, et al. Final results of a randomized, phase III study of rituximab with or without idelalisib followed by open-label idelalisib in patients with relapsed chronic lymphocytic leukemia. J Clin Oncol. 2019;37(16):1391-1402. doi:10.1200/JCO.18.01460

57. Patel VM, Balakrishnan K, Douglas M, et al. Duvelisib treatment is associated with altered expression of apoptotic regulators that helps in sensitization of chronic lymphocytic leukemia cells to venetoclax (ABT-199). Leukemia. 2017;31(9):1872-1881. doi:10.1038/ leu.2016.382

58. Roeker LE, Mato AR. Approaches for relapsed CLL after chemotherapy-free frontline regimens. Hematology. 2020;2020 (1):10-17. doi:10.1182/hematology.2020000168

59. Mato AR, Roeker LE, Jacobs R, et al. Assessment of the efficacy of therapies following venetoclax discontinuation in CLL reveals BTK inhibition as an effective strategy. Clin Cancer Res. 2020;26 (14):3589-3596. doi:10.1158/1078-0432.CCR-19-3815

60. Liu N, Rowley BR, Bull CO, et al. BAY 80-6946 is a highly selective intravenous PI3K inhibitor with potent $\mathrm{p} 110 \alpha$ and $\mathrm{p} 110 \delta$ activities in tumor cell lines and xenograft models. Mol Cancer Ther. 2013;12 (11):2319-2330. doi:10.1158/1535-7163.MCT-12-0993-T

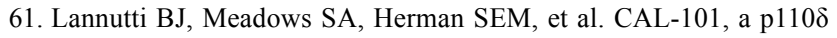
selective phosphatidylinositol-3-kinase inhibitor for the treatment of B-cell malignancies, inhibits PI3K signaling and cellular viability. Blood. 2011;117(2):591-594. doi:10.1182/blood-2010-03275305

62. Burris HA, Patel MR, Lanasa MC, et al. Activity of TGR-1202, a novel once-daily $\mathrm{PI} 3 \mathrm{~K} \delta$ inhibitor, in patients with relapsed or refractory hematologic malignancies. $J$ Clin Oncol. 2014;32 (15_suppl):2513. doi:10.1200/jco.2014.32.15_suppl.2513

63. Lampson BL, Brown JR. PI3K $\delta$-selective and PI $3 \mathrm{~K} \alpha / \delta$-combinatorial inhibitors in clinical development for B-cell non-hodgkin lymphoma. Expert Opin Investig Drugs. 2017;26(11):1267-1279. doi:10.1080/ 13543784.2017 .1384815
OncoTargets and Therapy

\section{Publish your work in this journal}

OncoTargets and Therapy is an international, peer-reviewed, open access journal focusing on the pathological basis of all cancers, potential targets for therapy and treatment protocols employed to improve the management of cancer patients. The journal also focuses on the impact of management programs and new therapeutic

Submit your manuscript here: https://www.dovepress.com/oncotargets-and-therapy-journa agents and protocols on patient perspectives such as quality of life, adherence and satisfaction. The manuscript management system is completely online and includes a very quick and fair peer-review system, which is all easy to use. Visit http://www.dovepress.com/ testimonials.php to read real quotes from published authors. 